Fast alle Erwachsene wünschen (nicht nur den eigenen) Kindern nur das Beste. Es gibt jedoch Fälle, in denen Jugendämter (JA) das Kindeswohl gefährdet sehen und nicht selten ist zu fragen, warum Jugendamtsmitarbeiter eine Gefahr sehen, die andere Einrichtungen nicht erkennen. Neben dem Jugendamt arbeiten andere Institutionen wie Kindergärten, Ärzte und Familienhilfen mit § 8a SGB VIII und müssen besondere Vorkommnisse einer möglichen Kindeswohlgefährdung melden.

Auch die Stadt Osnabrück, in der - entgegen dem Landestrend - die Zahl der Kindesinobhutnahmen gestiegen ist, scheint (insbesondere nach dem Kutzner-Fall) nicht dazu gelernt zu haben. Jugendamt, Sozialpädagogische Familienhilfe (SPFH), Adoptions- und Pflegekinderdienste sowie Verfahrensbeistände und Bereitschaftspflegefamilien scheinen den „Geist“ von Nienstedt und Westermann verinnerlicht zu haben. Die genannten Einrichtungen erwecken den Eindruck, eine Einheit, ein System zu bilden, das sich im Zweifel gegen die leiblichen Kindeseltern richtet.

\title{
Jugendämter und das Wohl von Kindern und Eltern
}

Es gibt in Osnabrück - und in ganz Deutschland - ein Strukturproblem. Notwendig ist eine Kontrollinstanz für Jugendämter. Schnellstens geboten ist die Unterstellung der Jugendämter unter die Fachaufsicht durch die Landesjugendämter, wie vor 1991. Auf kommunaler Ebene hilfreich sein kann die Einrichtung eines Beschwerdemanagements für Familien, die mit der Arbeit des Jugendamtes nicht einverstanden sind. Geboten ist - wie jetzt in Hamburg - die Überprüfung von (Bereitschafts-) Pflegeeltern. Leider sind wieder einmal neue Regelungen erst dann in Kraft getreten, als ein Kind zu Tode kam. Darüber hinaus könnte eine Beschränkung der Anzahl von Pflegekindern pro Familie sinnvoll sein. Ein Pflegekind pro Pflegeeltern, die nicht selten auch leibliche Kinder haben, erscheint angemessen.

Strukturell betrachtet sind insbesondere Jugendämter und SPFH wechselseitig voneinander abhängig. Das Jugendamt kann eine Familienhilfe anbieten bzw. gewähren, jedoch nicht selber durchführen. Freie Träger einer SPFH benötigen einen Auftrag durch ein Jugendamt.

Auch unter finanziellen Aspekten sind zwei Abhängigkeiten zu beobachten: So sind zum einen alle freien Träger einer SPFH sind vom Jugendamt / der Kommune abhängig, zum andern alle (Bereitschafts-) Pflegefamilien. Aus politikwissenschaftlicher Perspektive birgt so eine Konstellation das Risiko von „Gefälligkeitsberichten“, nach dem Motto: „Wessen Brot ich esse, dessen Lied singe ich“. Jeder einzelne Cent wird so zu einem Argument für das Verfassen von -sich zumindest teilweise widersprechenden- Berichten mit bestimmten, nicht selten für die leiblichen Eltern negativen Inhalten. Skandalös wäre es dann, wenn Gerichte nicht zur Aufklärung des Sachverhalts beitragen.

Inobhutnahmen, die dem Kindeswohl dienen sollen, können in bestimmten Fällen selbst eine Kindeswohlgefährdung darstellen. Daher ist die sorgfältige und begründete Abschätzung des Gefährdungsrisikos durch Jugendämter bzw. zuständige Familiengerichte eine verbindliche Verfahrensvorschrift und ist als solche in § 8a SGB VIII geregelt. Diese Vorschrift wird offenbar von zu wenig Jugendamtmitarbeitern und Gerichten beachtet.

Seit Bestehen der Jugendämter hat sich deren Selbstverständnis gewandelt. Auch sind finanzielle und personelle Rahmenbedingungen verändert worden. Mittlerweile scheinen Mitarbeiter von Jugendämtern heillos überfordert zu sein und greifen vermutlich aus reinem Selbstschutz zum nicht selten zu harten Mittel der Inobhutnahme - insbesondere bei Kindern mit Migrationshintergrund. Der Einwand, Eltern könnten ihre Rechte über Familiengerichte durchsetzen, klingt zynisch. Nicht wenige Betroffene und kritische Interessierte sehen daher in den mit viel Macht ausgestatteten Jugendämtern eine „neue Stasi-Behörde“ oder vergleichen sie mit dem nationalsozialistischen „Lebensborn-Verein“. Kluge Menschen wissen: Kinder sind Zukunft. Deren Wohlergehen - nicht das von erwachsenen Jugendamtsmitarbeitern - muss man im Auge haben. 